\title{
Cooling a Single Atom in an Optical Tweezer to Its Quantum Ground State
}

\author{
A. M. Kaufman, B. J. Lester, and C. A. Regal* \\ JILA, University of Colorado and National Institute of Standards and Technology, Boulder, Colorado 80309, USA \\ Department of Physics, University of Colorado, Boulder, Colorado 80309, USA
}

(Received 20 September 2012; published 29 November 2012)

\begin{abstract}
We report cooling of a single neutral atom to its three-dimensional vibrational ground state in an optical tweezer. After employing Raman sideband cooling for tens of milliseconds, we measure via sideband spectroscopy a three-dimensional ground-state occupation of about $90 \%$. We further observe coherent control of the spin and motional state of the trapped atom. Our demonstration shows that an optical tweezer, formed simply by a tightly focused beam of light, creates sufficient confinement for efficient sideband cooling. This source of ground-state neutral atoms will be instrumental in numerous quantum simulation and logic applications that require a versatile platform for storing and manipulating ultracold single neutral atoms. For example, these results will improve current optical-tweezer experiments studying atom-photon coupling and Rydberg quantum logic gates, and could provide new opportunities such as rapid production of single dipolar molecules or quantum simulation in tweezer arrays.
\end{abstract}

DOI: 10.1103/PhysRevX.2.041014

Subject Areas: Atomic and Molecular Physics, Quantum Physics, Quantum Information

Trapped ultracold neutral atoms provide a promising starting point for quantum simulation and computation. Ideally, experiments would be able to initialize a homogeneous array of atoms in an arbitrary quantum state, reconfigure this array in real time, and turn interactions on and off. Optical tweezers represent an interesting route toward this vision because they have the capability to integrate multiqubit storage, readout, and transport [1-4]. Recently, an idea for near-deterministic loading of optical-tweezer traps has been demonstrated [5]. By combining this idea with the capability to detect and correct occupation defects, one has a near-term possibility of building an ordered neutral-atom array atom by atom [6]. However, an unrealized prerequisite for this vision of a low-entropy tweezer array is the ability to place a specific single atom in its vibrational ground state. Such control of single-particle motion has been an integral part of trapped-ion experiments for decades. In this article, we show that analogous control can be realized with a neutral atom. We optically cool a single ${ }^{87} \mathrm{Rb}$ atom in a tweezer to its three-dimensional vibrational ground state with $90 \%$ probability, which is an improvement of 2 orders of magnitude over previous experiments employing laser-cooled atoms in optical tweezers.

Motional control of neutral atoms has a rich history, and increasingly interest has turned to the problem of singleatom control. To date, optical lattices created by standing waves of light have been the main platform for realizing motional control of collections of single neutral atoms.

\footnotetext{
*regal@jila.colorado.edu
}

Published by the American Physical Society under the terms of the Creative Commons Attribution 3.0 License. Further distribution of this work must maintain attribution to the author(s) and the published article's title, journal citation, and DOI.
Approaches have included dramatic demonstrations of the superfluid-Mott insulator transition of an evaporatively cooled gas [7,8] and exploration of the laser cooling of collections of atoms in a lattice [9-17]. Most recently, in spin-dependent lattices, experimenters have harnessed microwave signals to cool and control atomic motion [15,17]. Our work with an optical tweezer breaks with typical lattice experiments and instead more closely resembles the sideband cooling and spectroscopy techniques used with atomic ions [18]. We hold a single atom in a fardetuned tweezer trap and apply near-resonant, pulsed cooling and spectroscopy light that couples two ground-state hyperfine levels [Fig. 1(a)]. The complete separation of the trapping and cooling beams $[12,14,16]$ allows us to realize rapid cooling as well as low rates of atomic spontaneous emission due to the trapping light and hence long qubit coherence times.

Already, optical tweezers have been used to realize Rydberg quantum logic gates and a variety of protocols coupling atoms and photons. In these experiments, the thermal motion of the atoms has caused deleterious effects such as dynamic light shifts, mitigated atom-photon coupling, and dephasing of high-fidelity Rydberg gates $[3,4,19,20]$. Attaining full three-dimensional motional control [21,22] would not only strengthen current tweezer applications but also expand their use to experiments that are currently considered only in the context of evaporatively cooled gases. For example, one could combine two traps and realize significant wave-function overlap for Feshbach molecule association and hence creation and control of single dipolar molecules [21,23]. Or, with sufficient tunneling between traps and control of a spin degree of freedom, one could study strongly correlated physics proposed in lattices [24] with a platform amenable to studying nonequilibrium dynamics. 

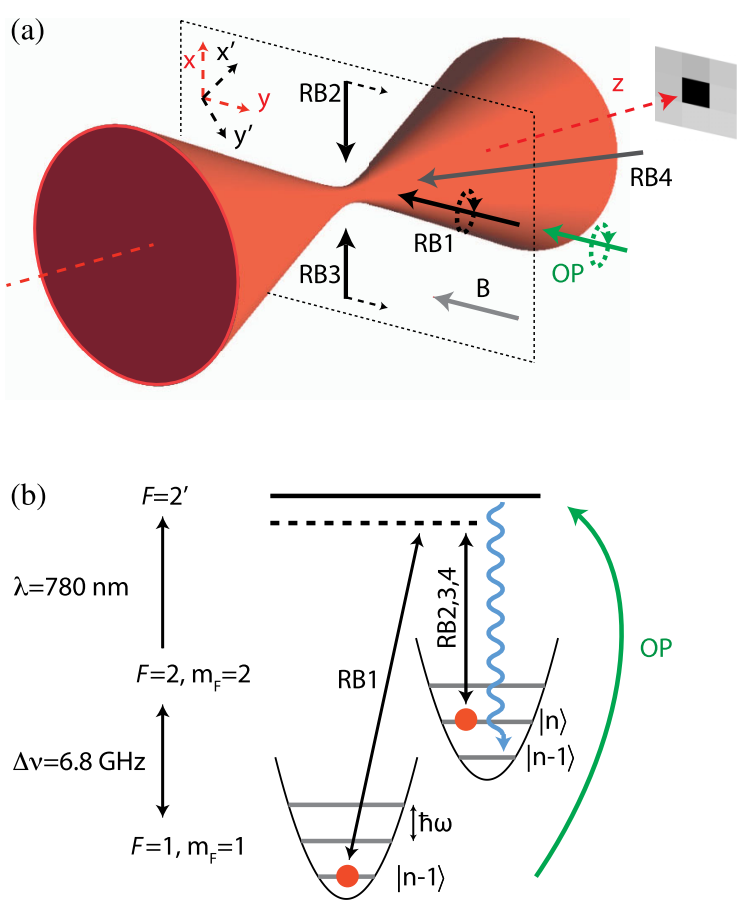

FIG. 1. Experimental setup for tweezer trap, detection, and three-dimensional motional control. (a) An optical tweezer created with a high-NA objective lens traps a single neutral atom, and the atom is imaged along the $z$ axis with the same lens. Orthogonal radial axes, indicated by $x^{\prime}$ and $y^{\prime}$, are addressed by Raman beam 1 (RB1) $\left(\sigma^{+}\right.$polarized) and RB2 ( $\pi$ polarized), or $\mathrm{RB} 1$ and RB3 ( $\pi$ polarized). RB1 and RB4 (linearly polarized in the $y-z$ plane) address the axial direction. Note that we should be able to cool vibrational motion along all three axes with a single pair of counterpropagating beams. (b) Level diagram for ${ }^{87} \mathrm{Rb}$ with associated beams from (a). The Raman light is $50 \mathrm{GHz}$ red, detuned of the excited state manifold. Optical pumping (OP) consists of the repump light on the $F=1 \rightarrow 2^{\prime}$ transition along with the optical-pumping light on $F=2 \rightarrow 2^{\prime}$.

The versatility of our tweezer-based trapping and cooling platform also presents new opportunities. In particular, a frontier in quantum interfaces is realizing strong coupling of neutral atoms to nanophotonic circuits [25-27], but trapping and controlling atoms in nanoscale, near-surface potentials remains a considerable challenge. Ground-state cooling of an atom in an optical tweezer advances this frontier by (1) providing a mobile, highly localized reservoir of single neutral atoms that can be more easily transferred to nanoscale potentials and (2) advancing techniques for in-situ cooling within these potentials.

We laser cool a trapped neutral atom to its ground state by employing a technique known as Raman sideband cooling [28,29]. Raman sideband cooling operates by iterating on a two-step process that manipulates the atomic spin and motion. It resolves motional transitions even when the trap frequency is less than the electronic-transition linewidth. In our scheme, we utilize two hyperfine levels of ${ }^{87} \mathrm{Rb}:\left|F, m_{F}\right\rangle \equiv|2,2\rangle$ and $|1,1\rangle$ [Fig. 1(b)], where $F$ is the hyperfine angular-momentum quantum number and
$m_{F}$ its projection. The first step is a stimulated Raman transition on $|2,2 ; n\rangle \leftrightarrow|1,1 ; n-1\rangle$ that reduces the vibrational state by one quantum while also realizing a spin flip. The second step is the dissipative step: The atom is optically pumped back to the initial spin state via a spontaneous Raman process, while the photon carries away entropy. Under a sequence of repetitions of these steps, population accumulates in the $|2,2 ; 0\rangle$ state because it is dark to the Raman beams and the optical pumping light.

For Raman cooling to be successful, the opticalpumping step must preserve the reduced vibrational state. The vibrational-excitation probability depends on the vibrational quantum number $n$ and the Lamb-Dicke parameter, $\eta^{\mathrm{OP}} \equiv x_{0} k$, where $x_{0}=(\hbar / 2 m \omega)^{1 / 2}$ is the oscillator length for a particle of mass $m$ and $k$ is the wave number associated with the optical pumping light. Raman cooling begins with an atom in a mixed thermal state, with a temperature corresponding to an average vibrational quantum number $\bar{n}$. In this case, the excitation probability due to a single scattered photon scales with $\left(\eta_{\mathrm{eff}}^{\mathrm{OP}}\right)^{2} \equiv$ $(2 \bar{n}+1)\left(\eta^{\mathrm{OP}}\right)^{2}$, and hence the Raman cooling efficiency scales inversely with $\left(\eta_{\text {eff }}^{\text {OP }}\right)^{2}$.

Therefore, ground-state cooling (achieving $\bar{n}=0$ ) requires (1) low enough initial temperatures before Raman cooling is initiated, and (2) sufficient confinement, i.e., large trap frequencies. To realize low initial temperatures in our optical-tweezer trap, we carefully apply the subDoppler cooling technique of polarization-gradient cooling (PGC) [30]. To realize strong three-dimensional confinement, we use a tightly focused optical-tweezer trap; the trap is formed using far-off-resonant light at $852 \mathrm{~nm}$ and a 0.6 numerical-aperture (NA) objective. With $2.8 \mathrm{~mW}$ of power in the central focal spot, we measure trap frequencies of $\left\{\omega_{z}, \omega_{x^{\prime}}, \omega_{y^{\prime}}\right\} / 2 \pi=\{30,154,150\} \mathrm{kHz}$. The large range of frequencies spanned by the axial $(z)$ and radial $(x, y)$ dimensions of our trap allow us to explore the challenges to robust Raman cooling that accompany increasing Lamb-Dicke parameters and initial occupations.

Another challenge specific to the optical-tweezer platform is effective magnetic fields (vector light shifts) induced by a linearly polarized dipole trap with a nonparaxial focus. This additional field could (1) dephase field-sensitive transitions, (2) disrupt the quantization axis and hence optical-pumping fidelity, and (3) increase realizable PGC temperatures. For our NA, detuning, and typical intensity, we calculate that the effective magnetic field is $\pm 0.13 \mathrm{G}$ over $\pm 50 \mathrm{~nm}$, and points along the crossproduct of the dipole trap axis $(z)$ and its polarization $(y)$ [31]. During Raman cooling, we set our quantization field to $3 \mathrm{G}$ in a direction orthogonal to the effective magnetic field, mitigating effects (1) and (2).

Our experiment begins by loading the tweezer trap from a magneto-optical trap overlapped with the opticaltrap focus. We use light-assisted collisions to realize zero or one atom in the trap with approximately equal 

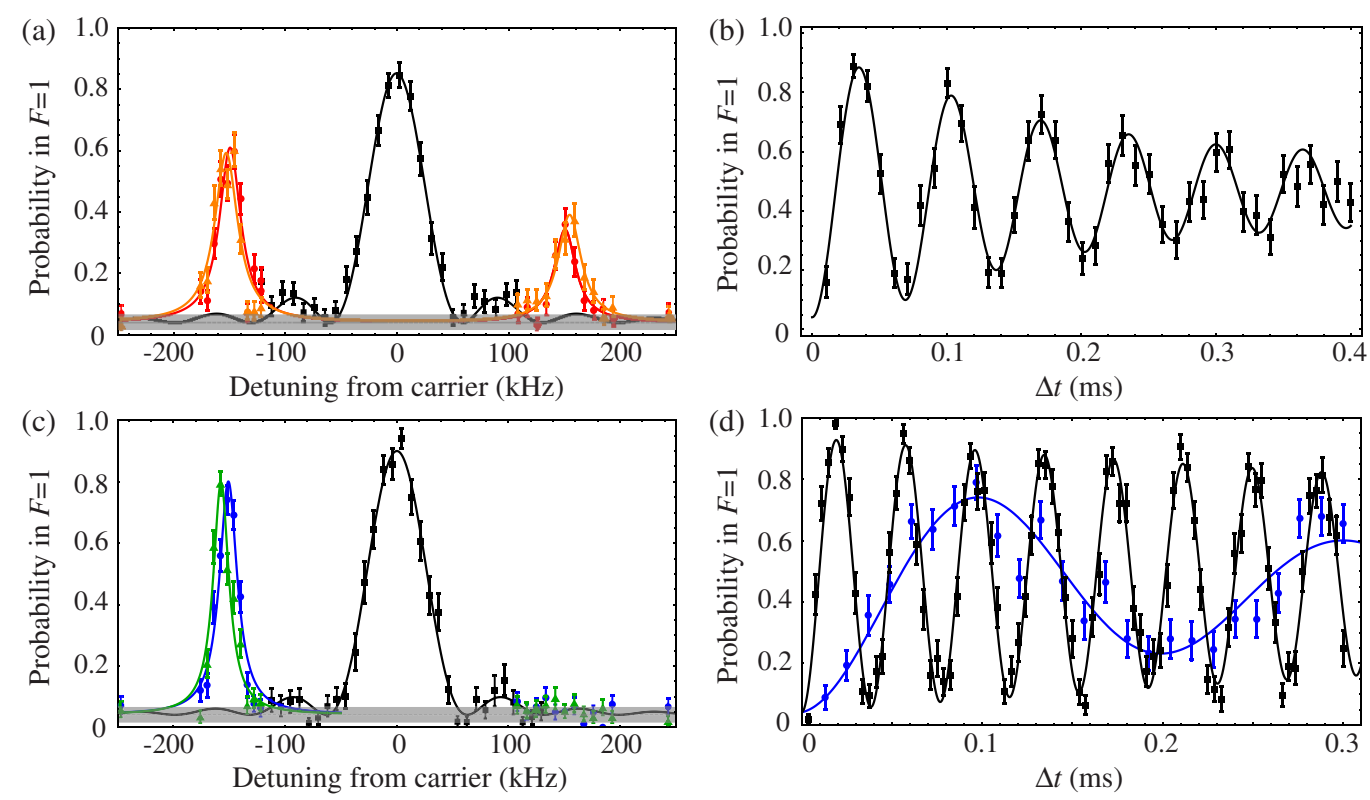

FIG. 2. Single-atom sideband spectra and Rabi oscillations in the radial dimensions before (a),(b) and after (c),(d) ground-state cooling. (a) The black squares are a carrier peak in the $y^{\prime}$ direction using a $\Delta t=15-\mu$ s (near- $\pi$ ) pulse. The red circles (orange triangles) are sidebands along the $y^{\prime}\left(x^{\prime}\right)$ axis for a $75-\mu$ s (near- $\pi$ ) pulse, demonstrating an initial thermal population of vibrational states. The solid lines are fits to a Rabi sinc function (defined in Supplemental Material, Ref. [35]) for the carrier and Lorentzians (an approximation) for the sidebands; each fit contains an offset at our measured background (gray shaded region centered at 0.04). (b) Carrier Rabi oscillations for the $y^{\prime}$ direction showing dephasing of a thermal state. Here, the carrier Rabi frequency is set to $15 \mathrm{kHz}$, instead of the usual $26 \mathrm{kHz}$. The solid line is a fit to the data using a thermal distribution of Rabi frequencies. (c) Raman-cooled radial sidebands; no Raman cooling is applied to the axial direction for these data. The black squares are a cooled carrier peak using a 15- $\mu$ s pulse. The blue circles (green triangles) are spectra along the $y^{\prime}\left(x^{\prime}\right)$ axis using a $75-\mu$ s pulse, displaying a significant asymmetry that is the hallmark of a large ground-state population. (d) Rabi oscillations for a radial ground-state cooled atom with a fit to a damped sine for the carrier (black squares) and the $\Delta n=+1$ sideband (blue circles), which demonstrates coherent control of the spin-motional states; the carrier dephasing is suppressed due to the purity of the vibrational distribution. Each data point is an average of 150 experimental runs, and hence approximately 75 atoms.

probability [1]. In order to postselect on the presence of one atom, we take an initial image of the atom immediately after the loading sequence. We start the cooling sequence by applying PGC light for $5 \mathrm{~ms}$ in a $\sigma^{+}-\sigma^{-}$configuration using three retro-reflected beams (see the Appendix). We address the challenging problem of stabilizing the phase of the interference pattern with respect to the subwavelength extent of the atom in the tweezer trap by modulating the position of our retro-reflecting mirrors at $1 \mathrm{kHz}$. This modulation yields a time-averaged cooling that does not exhibit shot-to-shot variations in the final PGC temperature due to slow fluctuations in the interference pattern.

As our first application of Raman coupling, we diagnose a thermal occupation using sideband spectroscopy. After PGC is performed, we optically pump the atom to $\left|F, m_{F}\right\rangle \equiv|2,2\rangle$. A single pair of Raman beams is chosen according to the dimension we wish to probe [Fig. 1(a)], and we interrogate the atom with a square pulse of length $\Delta t$, leading to transitions to the $|1,1\rangle$ state when a resonance is satisfied. The Lamb-Dicke parameter for the Raman transition, which quantifies the motional coupling, is given by $\eta^{\mathrm{R}} \equiv x_{0} \Delta k$, where $\Delta k$ is the momentum transferred by the Raman process along the motional axis. For our geometry, $\eta^{\mathrm{R}}=0.22$ for the radial dimension and $\eta^{\mathrm{R}}=0.23$ for the axial dimension.

Figure 2(a) shows sideband spectra after PGC along orthogonal radial directions. The asymmetry between the $\Delta n=-1$ and the $\Delta n=+1$ peaks determines the population of the atoms in the ground state. (Because we begin in the upper hyperfine state, the $\Delta n=-1$ peak is on the right in Fig. 2.) By equating the ratio of the $\Delta n=-1$ and $\Delta n=+1$ sidebands to $\bar{n} /(\bar{n}+1)$, we find $\bar{n}_{y^{\prime}}=1.1 \pm 0.4$ or $\mathrm{T}_{y^{\prime}}=11 \pm 3 \mu \mathrm{K}$, assuming a thermal population distribution; correspondingly, $\bar{n}_{x^{\prime}}=1.7 \pm 0.7$. Using the dephasing of the carrier Rabi oscillations in these data, we can also extract a temperature via the coherent evolution of the thermal state [Fig. 2(b)] to find $\mathrm{T}_{y^{\prime}} \leq 16 \pm 2 \mu \mathrm{K}$ [32]. For comparison, we also employ a standard thermometry technique in which the atom is quickly released, and the probability of recapturing the atom at a variable time later is measured and compared to a classical Monte Carlo model [33] from which we estimate a temperature of $13 \pm 1 \mu \mathrm{K}$. The agreement between our three measurements validates sideband spectroscopy as a reliable form of thermometry in an optical tweezer, and we find that low 
PGC temperatures are possible despite the varying effective magnetic fields within the focus.

For the radial dimensions, the PGC allows us to start our Raman cooling reasonably far into the Lamb-Dicke regime with $\eta_{\mathrm{eff}}^{\mathrm{OP}} \sim 0.3\left(\bar{n} \sim 1.5\right.$ and $\left.\eta^{\mathrm{OP}}=0.16\right)$. Like ion experiments, our cooling scheme operates in a pulsed format: We apply a coherent Raman transition on the $\Delta n=-1$ sideband using an intensity corresponding to a groundstate carrier Rabi frequency of $\Omega_{c}=2 \pi \times 26 \mathrm{kHz}$, where the $\Delta n=-1$ sideband Rabi frequency is $\Omega_{\mathrm{sb}} \sim \eta^{\mathrm{R}} \Omega_{c} \sqrt{n}$. Therefore, we apply $60-\mu$ s pulses for 47 cycles and switch to $75-\mu$ s pulses for the last three cycles when the remaining excited-state fraction is primarily in the first excited state. The pulses are applied to each radial axis by alternating Raman beam pairs. Interspersed between the Raman pulses are optical-pumping pulses $90 \mu$ s long that recycle the atom back to $|2,2\rangle$. Note that we do observe cooling of both radial dimensions even if we employ a single pair of cooling beams, indicating there is coupling between the radial dimensions of our anharmonic potential. To assure and verify cooling in both dimensions, however, we cool and probe the motion of the atom along each axis separately.

After Raman cooling, we see a significant asymmetry in the radial sideband spectroscopy due to a large groundstate occupation [Fig. 2(c)]. While the $\Delta n=-1$ sideband is suppressed, the $\Delta n=+1$ sideband has increased in height due to decreased dephasing as the thermal distribution is narrowed. Figure 2(d) shows Rabi oscillations for both the carrier (black squares) and $\Delta n=+1$ sideband (blue circles) transitions, the latter of which oscillates more slowly by a factor of $\eta^{\mathrm{R}}$. Figure 2(d) further demonstrates the coherence of our vibrational transitions. The carrier decays less quickly than in Fig. 2(b), as expected for colder atoms; the sideband transition decays slightly faster than the cold carrier due to its relative narrowness [34], but note that it maintains high contrast on the first oscillation. To assess our final occupation, we compare the measured signal at the position of the $\Delta n=-1$ transition peak to the measured background level due to any atoms left in $F=1$ and imperfect pushout efficiency. We find $\left\{\bar{n}_{x^{\prime}}, \bar{n}_{y^{\prime}}\right\}=$ $\left\{0.05_{-0.04}^{+0.05}, 0.02_{-0.02}^{+0.04}\right\}$.

To achieve large three-dimensional ground-state occupation, we must cool the atom along the more weakly confining, axial dimension of the trap where both spectroscopy and cooling are more challenging due to the smaller trap frequency. Figure 3(a) shows a thermal axial-mode spectrum after PGC. The near equality of the $\Delta n=+1$ and $\Delta n=-1$ transitions and the presence of significant second-order sidebands (given $\eta^{\mathrm{R}}=0.23$ for this dimension) are consistent with a small initial ground-state population. Assuming an isotropic initial temperature of $12 \mu \mathrm{K}$, we would expect $\bar{n}=8$, which corresponds to a challenging starting point of $\eta_{\mathrm{eff}}^{\mathrm{OP}}=1.4$. Further, the smaller trap frequency makes it difficult to spectroscopically separate the carrier and sideband peaks while maintaining Rabi frequencies that are insensitive to technical dephasing. For the cooling, a large Rabi frequency leads to off-resonant carrier transitions that cause heating, while too small a Rabi frequency leads to smaller transfer efficiencies and slow cooling.

Despite these barriers, we are able to Raman cool the axial motion and achieve the significant threedimensional ground-state occupations evidenced in Fig. 3(b) after $33 \mathrm{~ms}$ of cooling. (See the Appendix for pulse parameters.) Unlike the radial directions, we use different parameters for the axial cooling than are ideal for spectroscopy. To highlight this distinction, we show two spectra of cooled atoms. The axial mode spectrum in the center of Fig. 3(b) approximates the parameters used during our cooling, which balance speed with spectroscopic resolution. While there is a clear
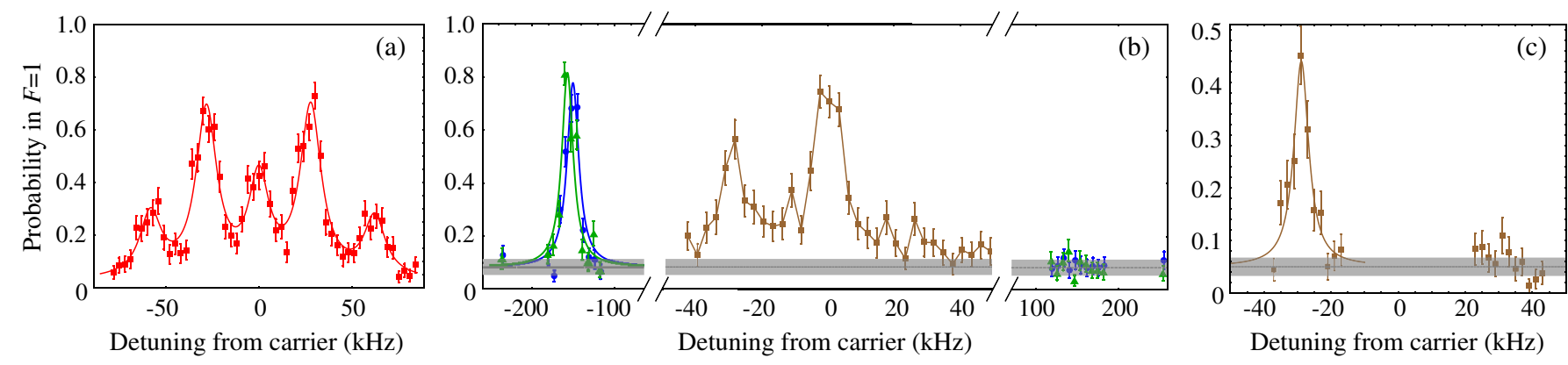

FIG. 3. Axial spectra and 3D ground-state cooling of a single neutral atom. (a) A thermal axial spectrum (red squares) using an intensity corresponding to a cold-carrier Rabi frequency of $12 \mathrm{kHz}$ and a $\Delta t=65-\mu$ s Raman pulse. The data are fit to Lorentzians (solid line) to guide the eye. (b) Result of simultaneous sideband cooling in three dimensions, demonstrating significant sideband asymmetries and simultaneous ground-state occupations in all dimensions. The axial data (center) illustrate our cooling parameters, and are obtained with a carrier Rabi frequency of $10.6 \mathrm{kHz}$ and a pulse of $236 \mu$ s (near- $\pi$ pulse on the ground-state $\Delta n=+1$ sideband, a $5 \pi$ pulse on the carrier). For the radial data, the blue circles (green triangles) are spectra along the $y^{\prime}\left(x^{\prime}\right)$ axis using a 75 - $\mu$ s pulse. The solid lines on the $\Delta n=+1$ sidebands are Lorentzian fits. (c) After 3D cooling, axial spectroscopy for a halved carrier Rabi frequency of $5 \mathrm{kHz}$ and a pulse of $450 \mu \mathrm{s}$. Better spectroscopic resolution affirms a large axial ground-state occupation. 
sideband asymmetry in the spectrum, the size of the Rabi frequency compared to the axial-trap frequency complicates a temperature analysis because off-resonant carrier transitions occur at the frequency position of the sidebands. In Ref. [35], we show calculated spectra for the axial pulse parameters used in Fig. 3(b), but a full comparison to this calculation is complicated by the partial dephasing we observe.

In Fig. 3(c), we halve the spectroscopy Rabi frequency to sacrifice coherence for spectroscopic resolution. For this Rabi frequency and pulse, the spectrum is dephased, and hence we can understand the spectrum simply as a set of multiple Lorentzians. We extract our axial temperature from the sideband asymmetry in Fig. 3(c), assuming that the dephasing uniformly affects the two sideband peaks. For both the radial and axial dimensions, we analyze the data attributing all of the signal observed at the $\Delta n=-1$ position to the sideband, thus placing an upper bound on the achieved temperature. Taking this approach, we extract temperatures in all three dimensions, again subtracting a measured background and assuming a thermal distribution. The result is $\left\{\bar{n}_{x^{\prime}}, \bar{n}_{y^{\prime}}, \bar{n}_{z}\right\}=$ $\left\{0.02_{-0.02}^{+0.07}, 0.01_{-0.01}^{+0.06}, 0.08_{-0.06}^{+0.08}\right\}$.

The above occupations indicate that we have cooled a single neutral atom to its ground state in an optical tweezer with $97_{-11}^{+3} \%$ probability in the radial plane, and $93_{-7}^{+5} \%$ probability in the axial direction, and hence a threedimensional ground-state population of $90_{-16}^{+8} \%$. While this estimate neglects imperfections in our spin preparation and detection, that is not a fundamental impediment to the final temperature. The current limitation to our quoted temperature is the precision of the spectroscopy measurement. Investigation of tunneling between adjacent tweezer traps would allow qualitatively new ways of analyzing the purity of the ground-state preparation, because bosonic enhancement of tunneling rates is contingent upon this preparation. Importantly, we have measured an upper bound to the heating rate of less than 1 radial vibrational quantum per second, suggesting that tunneling experiments are feasible with sufficiently tight, adjacent tweezer traps. Such experiments would open a new avenue of research for ultracold atoms in optical tweezers and establish their utility for investigations in several directions in quantum information and bottom-up approaches to quantum simulation.

We acknowledge T.P. Purdy and M. Feig for helpful input, and G. Downs for technical assistance. This work was supported by The David and Lucile Packard Foundation and the JILA NSF-PFC. C. R. acknowledges support from the Clare Boothe Luce Foundation and A.M.K. and B.J.L. from NDSEG and NSF-GRFP, respectively.

Note added-Related concurrent studies independently carried out by another group are described in Ref. [37].

\section{APPENDIX}

Optical-tweezer trap and single-atom loading. The optical tweezer is formed by a custom high-numericalobjective lens that is designed to be diffraction limited at both $850 \mathrm{~nm}$ and $780 \mathrm{~nm}$ [36]. From our measured trap frequencies, we infer a $1 / e^{2}$ beam radius of approximately $0.76 \mu \mathrm{m}$ and a depth of $1.4 \mathrm{mK}$ for our intensity. Aberrations dominated by astigmatism slightly increase our spot size compared to a diffraction-limited spot for a 0.6-NA lens. We measure a $1 / e$ lifetime of a single atom of about $5 \mathrm{~s}$, which can be extended at the expense of our trap load rate.

We load the tweezer trap from a vapor-cell magnetooptical trap (MOT) that has filled for 100-200 ms. The MOT consists of two orthogonal pairs of retro-reflected beams along $x^{\prime}$ and $y^{\prime}$, as well as a third pair at approximately $45^{\circ}$ to the $z$ axis and $10^{\circ}$ above the $y-z$ plane. For polarization-gradient cooling (PGC), the same beams are used as for the MOT light, and the bias fields are set for zero magnetic field; we zero our fields on the basis of microwave spectroscopy. The PGC detunings are optimized for each stage of the experiment. For loading into the tweezer trap, the $F=2-3^{\prime}$ light is detuned $60 \mathrm{MHz}$ red of the bare optical transition; the PGC to prepare for Raman cooling uses light $23 \mathrm{MHz}$ red of the bare optical transition [33].

All of our data rely on the ability to make spin-sensitive measurements of our ${ }^{87} \mathrm{Rb}$ atom. Our procedure is to apply light resonant with the light-shifted $F=2 \rightarrow 3^{\prime}$ transition while the atom is in the trap to retain atoms only in $F=1$. We then image the $F=1$ atoms by applying PGC (including repump) light. During the imaging, we collect fluorescence for 25 to $50 \mathrm{~ms}$ while imaging this light onto a CCD camera with about $7 \%$ overall efficiency with the same high-NA lens that creates the tweezer trap.

$3 D$ cooling parameters. For the three-dimensional cooling shown in Figs. 3(b) and 3(c), we toggle the Raman laser pulses to address each of the axes of the trap, and between each pulse we insert $90 \mu$ s of optical pumping. The cooling process in total occurs in 75 cycles: The first 50 cycles use a Raman pulse length of $\Delta t=$ $48 \mu \mathrm{s}(\Delta t=40 \mu \mathrm{s})$ for the radial (axial) directions, and then $\Delta t=72.5 \mu \mathrm{s}(\Delta t=80 \mu \mathrm{s})$ for the final 25 cycles. We use an intensity corresponding to a cold-carrier Rabi frequency of $13 \mathrm{kHz}$ for the axial direction, and $31 \mathrm{kHz}$ for the radial dimension.

[1] N. Schlosser, G. Reymond, I. Protsenko, and P. Grangier, Sub-Poissonian Loading of Single Atoms in a Microscopic Dipole Trap, Nature (London) 411, 1024 (2001).

[2] J. Beugnon, C. Tuchendler, H. Marion, A. Gaëtan, Y. Miroshnychenko, Y. R. P. Sortais, A. M. Lance, M. P. A. Jones, G. Messin, A. Browaeys, and P. Grangier, Two-Dimensional Transport and Transfer of a Single 
Atomic Qubit in Optical Tweezers, Nat. Phys. 3, 696 (2007).

[3] X. L. Zhang, L. Isenhower, A. T. Gill, T. G. Walker, and M. Saffman, Deterministic Entanglement of Two Neutral Atoms via Rydberg Blockade, Phys. Rev. A 82, 030306 (R) (2010).

[4] T. Wilk, A. Gaëtan, C. Evellin, J. Wolters, Y. Miroshnychenko, P. Grangier, and A. Browaeys, Entanglement of Two Individual Neutral Atoms Using Rydberg Blockade, Phys. Rev. Lett. 104, 101502 (2010).

[5] T. Grünzweig, A. Hilliard, M. McGovern, and M. F. Andersen, Near-Deterministic Preparation of a Single Atom in an Optical Microtrap, Nat. Phys. 6, 951 (2010).

[6] D. S. Weiss, J. Vala, A. Thapliyal, S. Myrgren, U. Vazirani, and K. Whaley, Another Way to Approach Zero Entropy for a Finite System of Atoms, Phys. Rev. A 70, 040302(R) (2004).

[7] W. S. Bakr, J. I. Gillen, A. Peng, S. Fölling, and M. Greiner, A Quantum Gas Microscope for Detecting Single Atoms in a Hubbard-Regime Optical Lattice, Nature (London) 462, 74 (2009).

[8] C. Weitenberg, M. Endres, J. F. Sherson, M. Cheneau, P. Schauß, T. Fukuhara, I. Bloch, and S. Kuhr, Single-Spin Addressing in an Atomic Mott Insulator, Nature (London) 471, 319 (2011).

[9] S. E. Hamann D. Haycock, G. Klose, P. Pax, I. Deutsch, and P. Jessen, Resolved-Sideband Raman Cooling to the Ground State of an Optical Lattice, Phys. Rev. Lett. 80, 4149 (1998).

[10] H. Perrin, A. Kuhn, I. Bouchoule, and C. Salomon, Sideband Cooling of Neutral Atoms in a Far-Detuned Optical Lattice, Europhys. Lett. 42, 395 (1998).

[11] V. Vuletić, C. Chin, A. J. Kerman, and S. Chu, Degenerate Raman Sideband Cooling of Trapped Cesium Atoms at Very High Atomic Densities, Phys. Rev. Lett. 81, 5768 (1998).

[12] D.-J. Han, S. Wolf, S. Oliver, C. McCormick, M. DePue, and D. Weiss, 3D Raman Sideband Cooling of Cesium Atoms at High Density, Phys. Rev. Lett. 85, 724 (2000).

[13] P. Maunz, T. Puppe, I. Schuster, N. Syassen, P. W. H. Pinkse, and G. Rempe, Cavity Cooling of a Single Atom, Nature (London) 428, 50 (2004).

[14] A. D. Boozer, A. Boca, R. Miller, T. E. Northup, and H. J. Kimble, Cooling to the Ground State of Axial Motion for One Atom Strongly Coupled to an Optical Cavity, Phys. Rev. Lett. 97, 083602 (2006).

[15] L. Förster, M. Karski, J.-M. Choi, A. Steffen, W. Alt, D. Meschede, A. Widera, E. Montano, J. Lee, W. Rakreungdet, and P. Jessen, Microwave Control of Atomic Motion in Optical Lattices, Phys. Rev. Lett. 103, 233001 (2009).

[16] S. Blatt, J. Thomsen, G. Campbell, A. Ludlow, M. Swallows, M. Martin, M. Boyd, and J. Ye, Rabi Spectroscopy and Excitation Inhomogeneity in a OneDimensional Optical Lattice Clock, Phys. Rev. A 80, 052703 (2009).

[17] X. Li, T. A. Corcovilos, Y. Wang, and D. S. Weiss, 3D Projection Sideband Cooling, Phys. Rev. Lett. 108, 103001 (2012).
[18] C. Monroe, D. Meekhof, B. King, S. Jefferts, W. Itano, D. Wineland, and P. Gould, Resolved-Sideband Raman Cooling of a Bound Atom to the 3D Zero-Point Energy, Phys. Rev. Lett. 75, 4011 (1995).

[19] B. Darquié, M. P. A. Jones, J. Dingjan, J. Beugnon, S. Bergamini, Y. Sortais, G. Messin, A. Browaeys, and P. Grangier, Controlled Single-Photon Emission from a Single Trapped Two-Level Atom, Science 309, 454 (2005).

[20] M. K. Tey, Z. Chen, S. A. Aljunid, B. Chng, F. Huber, G. Maslennikov, and C. Kurtsiefer, Strong Interaction Between Light and a Single Trapped Atom without the Need for a Cavity, Nat. Phys. 4, 924 (2008).

[21] F. Serwane, G. Zurn, T. Lompe, T. B. Ottenstein, A. N. Wenz, and S. Jochim, Deterministic Preparation of a Tunable Few-Fermion System, Science 332, 336 (2011).

[22] B. Zimmermann, T. Müller, J. Meineke, T. Esslinger, and H. Moritz, High-Resolution Imaging of Ultracold Fermions in Microscopically Tailored Optical Potentials, New J. Phys. 13, 043007 (2011).

[23] K.-K. Ni, S. Ospelkaus, M. H. G. de Miranda, A. Pe'er, B. Neyenhuis, J. J. Zirbel, S. Kotochigova, P. S. Julienne, D. S. Jin, and J. Ye, A High Phase-Space-Density Gas of Polar Molecules, Science 322, 231 (2008).

[24] B. Paredes and I. Bloch, Minimum Instances of Topological Matter in an Optical Plaquette, Phys. Rev. A 77, 023603 (2008).

[25] T. Aoki, B. Dayan, E. Wilcut, W. P. Bowen, A. S. Parkins, T. J. Kippenberg, K. J. Vahala, and H.J. Kimble, Observation of Strong Coupling between One Atom and a Monolithic Microresonator, Nature (London) 443, 671 (2006).

[26] E. Vetsch, D. Reitz, G. Sagué, R. Schmidt, S. T. Dawkins, and A. Rauschenbeutel, Optical Interface Created by Laser-Cooled Atoms Trapped in the Evanescent Field Surrounding an Optical Nanofiber, Phys. Rev. Lett. 104, 203603 (2010).

[27] D. E. Chang, J. Thompson, H. Park, V. Vuletić, A. Zibrov, P. Zoller, and M. Lukin, Trapping and Manipulation of Isolated Atoms Using Nanoscale Plasmonic Structures, Phys. Rev. Lett. 103, 123004 (2009).

[28] A. J. Kerman, Ph.D. thesis, Stanford University, 2002.

[29] D. Leibfried, R. Blatt, C. Monroe, and D. Wineland, Quantum Dynamics of Single Trapped Ions, Rev. Mod. Phys. 75, 281 (2003).

[30] P. Lett, R. Watts, C. Westbrook, W. Phillips, P. Gould, and H. Metcalf, Observation of Atoms Laser Cooled below the Doppler Limit, Phys. Rev. Lett. 61, 169 (1988).

[31] B. Richards and E. Wolf, Electromagnetic Diffraction in Optical Systems. II. Structure of the Image Field in an Aplanatic System, Proc. R. Soc. A 253, 358 (1959).

[32] See Supplemental Material at http://link.aps.org/ supplemental/10.1103/PhysRevX.2.041014 for the thermal dephasing model.

[33] C. Tuchendler, A. M. Lance, A. Browaeys, Y. R. P. Sortais, and P. Grangier, Energy Distribution and Cooling of a Single Atom in an Optical Tweezer, Phys. Rev. A 78, 033425 (2008). 
[34] See Supplemental Material at http://link.aps.org/ supplemental/10.1103/PhysRevX.2.041014 for a discussion of the dephasing.

[35] See Supplemental Material at http://link.aps.org/ supplemental/10.1103/PhysRevX.2.041014 for a discussion of spectroscopy line shapes and a simulation of spectra with overlapping carrier and sideband transitions.
[36] See Supplemental Material at http://link.aps.org/ supplemental/10.1103/PhysRevX.2.041014 for a description of the lens and optical layout.

[37] J.D. Thompson, T. G. Tiecke, A. S. Zibrov, V. Vuletić, and M.D. Lukin, Coherence and Raman Sideband Cooling of a Single Atom in an Optical Tweezer, arXiv:1209.3028. 